\title{
Organspende am Lebensende
}

\author{
Alex Frei ${ }^{a}$, Urs Aemissegger ${ }^{a}$, Alois Beerli ${ }^{b}$, Marlène Sicher ${ }^{c}$, Georg Stoffel ${ }^{b}$ \\ ${ }^{a}$ Dr. med., Facharzt für Allgemeine Innere Medizin, Winterthur; ${ }^{b}$ Dr. med., Facharzt für Innere Medizin, Winterthur; ${ }^{c}$ Intensivpflegefachfrau, \\ Pflegeexpertin BScN, Zürich
}

\section{Leben ist unantastbar. Organ-Lebendspenden sollen erlaubt bleiben. Organspen- den am Lebensende sollten nicht mehr durchgeführt werden.}

\section{Beachten Sie zu diesem Thema auch das «Zu guter Letzt» von Samia Hurst auf S. 534 in dieser Ausgabe.}

Der besseren Lesbarkeit wegen wird die männliche Form verwendet. Frauen sind gleichermassen angesprochen.
Der Verein ÄPOL (Ärzte und Pflegefachpersonen gegen Organspende am Lebensende) ist auf Initiative von Ärzten aus den Bereichen Hausarztmedizin und Psychotherapie, sowie von Intensivpflegefachpersonen entstanden. Aufgrund persönlicher Erfahrungen und Überlegungen sind wir überzeugt, dass Organspenden am Lebensende nicht vertretbar sind. Wir wollen erreichen, dass keine Organspenden am Lebensende mehr durchgeführt werden. Lebendspenden sollen erlaubt bleiben.

\section{Der Ablauf von Organspenden}

Organspende nach Hirntod («Donation after Brain Death» [DBD]): Menschen mit Hirntod liegen auf Intensivstationen, sie werden beatmet, ihr Herz schlägt und ihre Haut ist rosig und warm. Sie sehen aus wie Schlafende. Nachdem der Tod des Hirns festgestellt wurde, werden sie für tot erklärt, womit sie zu Organspendern werden können.

\section{Résumé}

Les arguments suivants sont avancés pour interdire le don d'organe en fin de vie: 1) Seuls des organes vivants peuvent être transplantés. Les donneurs sont donc encore vivants lors du prélèvement, qui provoque leur décès. 2) La médecine de transplantation ne sait pas ce qui se passe au niveau de la conscience, ni au niveau psychique et intellectuel, mais fait comme si elle le savait. 3) La médecine de transplantation prélève des organes à des personnes en mort cérébrale. Cela signifie implicitement que seule la mort cérébrale est importante, mais que celle du corps et des autres organes ne compte pas dans le décès d'une personne. Cette hypothèse ne peut être prouvée. 4) La médecine de transplantation transplante des organes vivants. Elle intervient donc dans la vie - celle du donneur aussi - et il convient de prouver qu'elle ne porte pas préjudice à la vie des donneurs. Ce qui est impossible. 5) Le processus de mort n'est pas terminé au moment du prélèvement d'organes et est donc perturbé.

Nous rectifierons ensuite les informations trompeuses véhiculées dans I'opinion publique sur le concept de mort cérébrale et sa survenue et présenterons les conclusions actuelles des débats internationaux.

Von der Intensivstation werden sie, nachdem sich ihre Familie von ihnen verabschiedet hat, zur Explantation in den Operationssaal gebracht. Dort kam es früher beim Aufschneiden des Körpers manchmal zu Blutdruckanstieg, Herzrasen, Schwitzen, Tränenfluss und zu Bewegungen mit Armen und Beinen [1]. Das sind Reaktionen auf Schmerzen, die durch Rückenmarksreflexe gesteuert werden. Diese Schmerzreaktionen werden nach heutiger Lehrmeinung als bedeutungslos angesehen, weil das Zentrum des Nervensystems, das Hirn, ausgefallen ist und die Schmerzen daher nicht mehr wahrgenommen werden können. Dass Spender diese Schmerzen nicht spüren, ist aber wissenschaftlich nicht belegbar (Cavete collegae, Irrtum möglich!).

Damit sich Spender während der Explantation nicht bewegen, erhalten sie heute in der Schweiz eine Allgemeinanästhesie. In Deutschland hingegen werden nur muskellähmende Medikamente verabreicht.

Anschliessend folgt eine bis achtstündige Operation, während der verschiedene Ärzteteams inmitten aufwendigster Technik und Apparaturen ein Organ nach dem anderen entnehmen. Das Herz wird durch kalte Perfusionslösung gelähmt, so zum Stillstand gebracht und herausgeschnitten [2]. Am Ende der Operation wird der Körper zugenäht, gewaschen und Verbände werden angelegt. Die Angehörigen können ihn dann, wenn sie möchten, nochmals sehen.

Organspende nach Herztod («Donation after Circulatory Death» [DCD]): In der Schweiz, nicht aber in Deutschland, ist es erlaubt, fünf Minuten nach HerzKreislauf-Stillstand Organe zu entnehmen. Dabei werden schwerkranke Patienten mit aussichtsloser Prognose, auf Wunsch im Beisein der Angehörigen, in den Operationssaal gebracht, wo alle lebenserhaltenden Massnahmen gestoppt werden und gewartet wird, bis das Herz aufhört zu schlagen. Exakt fünf Minuten nach Herzstillstand, wenn der Hirntod eingetreten ist, werden die Angehörigen aus dem Operationssaal gebeten. Der Spender wird in der Folge sofort reintubiert, laparotomiert und die Explantation wird durchgeführt. 


\section{Fünf Gründe für das Verbot von Organspenden am Lebensende}

1. Organspender sind zum Zeitpunkt der Organentnahme nicht tot. Sie sind am Sterben und werden durch die Entnahme getötet.

Es können nur lebende Organe verpflanzt werden und diese können nur aus lebenden Körpern entnommen werden. Organspender sind bei der Organentnahme noch lebendig [3]. Die implizite Behauptung der Transplantationsmedizin, sie entnehme lebende Organe postmortal, also von Toten, ist in sich ein Widerspruch. Bei Menschen mit totem Hirn gibt es viele Lebenszeichen. Solange sie beatmet und ernährt werden, regulieren sie die Körpertemperatur, bekämpfen Infektionen, heilen Wunden. Hirntote Kinder wachsen und kommen in die Pubertät und schwangere hirntote Frauen können per Kaiserschnitt gesunde Kinder zur Welt bringen.

Wie umstritten der Hirntod als Todesdefinition ist, zeigt auch die Tatsache, dass im Deutschen Ethikrat 2015 insgesamt 7 von 26 Mitgliedern den Hirntod nicht als hinreichende Bedingung für den Tod eines Menschen bezeichneten [4].

Hirntote Organspender sterben statt im Kreise ihrer Angehörigen im Operationssaal inmitten aufwendigster Technik. Das ist eine traurige und für viele Menschen verstörende Vorstellung. Menschen dürfen Menschen nicht töten, auch nicht, um anderen Menschen zu helfen. Das ist ein massiver kultureller Bruch. Es darf kein wertvolles (Empfänger) und unwertes (Spender) Leben geben. Jedes menschliche Leben muss unantastbar sein.

Diese Begründung alleine müsste genügen, um Organtransplantationen am Lebensende zu verbieten.

2. Es gibt kein Wissen darüber, was beim Tod mit dem Bewusstsein, dem Geistig-Seelischen, geschieht.

Wir wissen nicht, ob das Bewusstsein, das Geistig-Seelische, mit dem Eintreten des Hirntodes erlischt, oder ob es auf eine für uns nicht erfassbare Weise weiterexistiert. Wir wissen auch nicht, ob das Geistig-Seelische, sofern es weiterexistiert, noch leiden kann. Dies alles aber müssten wir wissen, um sicher zu sein, dass Organtransplantationen am Lebensende Spendern nicht schaden. Dass die Transplantationsmedizin ohne dieses Wissen Organe transplantiert, ist unhaltbar.

3. Es gibt keine wissenschaftlichen Belege, dass nur das Sterben des Hirns und nicht auch das des Körpers und der übrigen Organe für das Sterben des Menschen bedeutsam ist.

Das Handeln der Transplantationsmedizin beruht auch aus diesem Blickwinkel nur auf Annahmen und nicht auf gesichertem Wissen. Damit verstösst es gegen die Regeln medizinisch-wissenschaftlichen Handelns.

Der ganze Körper des Menschen lebt und stirbt, nicht nur das Hirn. So spielt sich zum Beispiel das Gefühlsleben im Körper ab (im Bauch, Herzbereich, Hals, etc.) und nicht im Hirn. Auch beinhaltet der Körper mit dem enterischen Nervensystem, auch Bauchhirn genannt, annähernd gleichviele Nervenzellen, wie das Hirn im Kopf.

Der Mensch ist schlicht nicht erschöpfend erforscht. Die heutige Praxis von Organtransplantationen am Lebensende könnte sich in Zukunft als Fehler, als eine menschliche und wissenschaftliche Tragödie erweisen.

\section{Indem lebende Organe verpflanzt werden, wird ins} Leben, auch das der Spender, eingegriffen.

Mit dem Eingriff ins Leben der Spender entsteht die Möglichkeit, diesem Leben zu schaden. Daraus wiederum ergibt sich die Pflicht, nachzuweisen, dass Nutzen und Nebenwirkungen des Eingriffs für Spender in einem annehmbaren Verhältnis stehen, insbesondere, dass der Eingriff Spendern keinen unverhältnismässigen Schaden zufügt. Diesen Nachweis kann die Transplantationsmedizin nicht erbringen.

5. Der Sterbeprozess ist zum Zeitpunkt der Explantation noch nicht beendet und wird gestört.

Bei Menschen mit totem Hirn sind 3\% des Körpers tot, 97\% leben noch. Zudem dauert es nach Herzstillstand ungefähr sieben Tage, bis die letzten Zellen im Körper gestorben sind [5]. Der Sterbeprozess ist also bei der Explantation noch nicht abgeschlossen und wird gestört.

Die Transplantationsmedizin am Lebensende kann nicht nachweisen, dass sie Spendern durch die Explantation und die Störung des Sterbeprozesses nicht schadet. Damit verstösst sie gegen fundamentale Regeln des medizinisch-wissenschaftlichen Handelns und möglicherweise gegen das Gebot des Nicht-Schadens der ärztlichen Ethik (Primum nil nocere). Das ist in unseren Augen verantwortungslos.

Die Transplantationsmedizin lässt die Öffentlichkeit in einem falschen Glauben.

Heute muss davon ausgegangen werden, dass Menschen explantiert werden, die geglaubt haben, sie seien zum Zeitpunkt der Organentnahme eine Leiche im herkömmlichen Sinn: keine Atmung, kein Herzschlag, kalte, blasse Haut. Das Bundesamt für Gesundheit, Swisstransplant und die Medien betonen immer wieder, dass Organe «nach dem Tod» entnommen werden, 
ohne klar zu stellen, dass seit 2007 eine neue Todesdefinition gilt und dass es erhebliche Unterschiede zwischen einem Toten nach heutiger Definition und einer herkömmlichen Leiche gibt. Die Öffentlichkeit in diesem falschen Glauben zu lassen ist inakzeptabel.

ÄPOL fordert, dass, solange Organspenden am Lebensende erlaubt sind, Spender ihre Zustimmung zu Organspende gemäss dem heutigen Standard als informierte Entscheidung gefällt haben müssen. Jede Person mit einem Organspendeausweis muss den Unterschied zwischen einem hirntoten Menschen und einer herkömmlichen Leiche kennen.

Auch weltweit führende Mediziner und Ethiker sind zur Einsicht gelangt, dass Menschen mit totem Hirn nicht tot sind.

Mediziner und Ethiker um Robert Truog, dem jetzigen Direktor des Zentrums für Bioethik der Harvard Medical School, das 1968 das Hirntodkonzept lanciert hatte, sind heute der Ansicht, dass Menschen mit totem Hirn zwar nicht tot sind, dass es aber dennoch moralisch richtig ist, ihnen Organe zu entnehmen [6]. Sie weisen damit ebenfalls auf das Dilemma, das im Hirntodkonzept per se drinsteckt, hin und hoffen, dass die Öffentlichkeit ihre Ansicht in Zukunft teilt. Sie fordern eine Abkehr von der Dead Donor Rule (Toter-Spender-Regel) und eröffnen damit die Debatte des «Justified Killing» (gerechtfertigtes Töten).

Diese Debatte müsste, wenn an Organspenden am Lebensende festgehalten wird, auch in der Schweiz geführt werden.

Wie ist es möglich, dass seit 50 Jahren weltweit mit dem Hirntodkonzept eine Todesdefinition gilt, die derart umstritten ist?

Ein Ad-Hoc-Komitee der Harvard Medical School schlug 1968 den Hirntod als neue Todesdefinition vor, um erstens bei hirntoten Menschen ohne angeklagt zu werden die lebenserhaltenden Therapien stoppen $\mathrm{zu}$ können und um zweitens zu verhindern, dass "veraltete Todeskriterien zu Streit bei der Organbeschaffung für Transplantationen führen» [7]. In ihrem Bericht begründete das Komitee aber nicht, warum der Hirntod dem Gesamttod des Menschen entsprechen soll (!). Es vertrat lediglich die Ansicht, dass die herkömmliche Todesdefinition mit Herz- und Atemstillstand angesichts der Fortschritte der Intensivmedizin veraltet («obsolete») sei.
Dieser Vorschlag wurde weltweit sofort aufgenommen. Bereits 1968 wurden über 100 Herztransplantationen durchgeführt und damit Tatsachen geschaffen. Verschiedene Gremien versuchten später, eine schlüssige Begründung für die Gleichung «Hirntod gleich Gesamttod» nachzuliefern. Die erste Begründung lautete, dass Hirntote tot seien, da das Hirn das zentrale Steuerorgan des menschlichen Körpers sei und mit seinem Ausfall der Tod sofort eintrete. Diese Begründung wurde durch das jahrelange Überleben von Hirntoten widerlegt. Die neueste Begründung, 2008 formuliert durch den «President's Council on Bioethics» der USA lautet, dass Hirntote tot seien, «weil die Integration in die Umwelt fehle» [8]. Auch diese Begründung überzeugt jedoch nicht abschliessend. Die aus Sicht jedes potentiellen Organspenders und der ärztlichen Ethik vorrangige Frage, ob kein Schaden angerichtet wird, ob also Transplantationen Spendern nicht schaden könnten, wird nie gestellt und diskutiert.

Offenbar verdrängt die ethische Verpflichtung und Motivation, schwerkranken Menschen zu helfen, und das Streben nach medizinischem Fortschritt und Prestige die Einsicht, dass das Fundament der Transplantationsmedizin am Lebensende nicht tragfähig ist und dass nicht ausgeschlossen werden kann, dass die Transplantationsmedizin Organspendern Leid zufügt.

\section{Literatur}

1 Lubitsch A, Schwelm H. Anästhesie zur Multiorganentnahme, Abschlussarbeit in der Weiterbildung zur Pflegefachfrau für Anästhesie und Reanimation, Kurs 2005, Universitätsspital Basel (verlinkt auf unserer Homepage www.aepol.net unter Literatur).

2 Swisstransplant/CNDO The Swiss Donation Pathway, Modul VI, Organ- und Gewebeentnahme.

3 Kersting D. Tod des Körpers oder Tod der Person? Ethik Med. 2017;29:217-32.

4 Deutscher Ethikrat, Hirntod und Entscheidung zur Organspende, Stellungnahme, 24.2.2015

5 Marsch S. When is a person dead? Uni Nova - Research Magazine of the University of Basel 125/2015, pp 33-4

6 Aviv R. What Does it Mean to Die? The New Yorker 5.2.2018, p 25

7 Beecher H, Adams R, Barger C, Curran W, Denny-Brown D, Farnsworth D et al. A Definition of Irreversible Coma, JAMA, Aug 5, 1968, Vol 205, No 6

8 Controversies in the Determination of Death: A White Paper by the President's Council on Bioethics, Washington D.C. Dec 2008

Weitere Informationen finden Sie auf unserer Homepage www. aepol.net. Neue Mitglieder sind herzlich willkommen. 